\section{D PEROVSKITES}

\section{Stand up to stand out}

Nat. Commun. 9, 1336 (2018)

Two-dimensional (2D) Ruddlesden-Popper metal halide perovskites (MHPs) offer a promising solution to the notorious moisture sensitivity of bulk perovskites. However, in devices with vertical architecture, the insulating bulky ammonium cations that separate 2D perovskites slabs impede efficient out-of-plane charge transport. Now, Chen et al. have devised a strategy to enhance optoelectronic properties of 2D MHPs by controlling the alignment of vertically oriented layers.

The researchers used a two-step fabrication procedure involving solution processing and low-temperature annealing to achieve thin films with various degrees of molecular orientation. X-ray measurements reveal that nucleation and growth of $2 \mathrm{D}$ perovskites occur at the precursor liquid-air interface. The assumed orientation stems from the tendency of the aliphatic chain of butylammonium molecules to remain in the solution environment. By tuning the crystallization conditions, specifically by adding a pre-crystallization annealing step, Chen and co-workers were able to obtain thin films of vertically oriented $2 \mathrm{D}$ perovskites with near $100 \%$ degree of the out-of-plane preferential alignment. When made into a solar cell, such optimized 2D MHPs show a strong short-circuit current increase and an overall efficiency improvement in comparison with randomly oriented crystals.
QUANTUM COMMUNICATION

Telecom single photons thaw

Sci. Adv. 4, eaar3580 (2018)

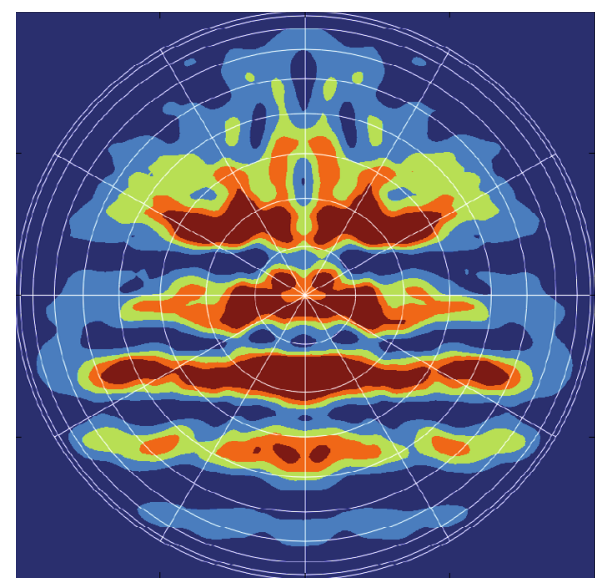

Credit: AAAS

Single-photon emitters (SPEs) are key components in quantum technology. Using single photons at telecom rather than ultraviolet-visible wavelengths could suppress attenuation losses in optical cables. However, such SPEs have so far been restricted to cryogenic temperatures. Now, Zhou et al. demonstrate single-photon emission at telecom wavelengths from gallium nitride $(\mathrm{GaN})$ defects that generate photons of high purity and brightness at room temperature.

The researchers use a $950 \mathrm{~nm}$ diode laser to excite the defects in GaN single-crystal films deposited on a sapphire substrate and observe single-photon emission with wavelengths between 1,085 and 1,340 nm. Though the room-temperature emission was
CARBON-WATER DEVICE

\section{Energy from quiescent water}

Energy harvesting from water is of significant interest due to the abundance and sustainability of this natural resource. However, flowing water is frequently required for energy converters to generate large amounts of electricity. Now, Peng and co-workers report a power generation system based on carbon materials that can efficiently harvest electricity from stationary pure water.

First, oxygen-modulated carbon nanotube (OCNT) yarns are prepared by an oxygen microplasma treatment. The oxygen modulated $\mathrm{sp}^{2}$-hybridized carbon systems exhibit stronger surface polarization than the pristine CNT (PCNT) yarns, as evidenced by X-ray adsorption studies. Then, the OCNT yarns are connected to PCNT yarns and immersed in quiescent water. The device works like a battery, in which the OCNT yarns, PCNT yarns and pure water act as the cathode, the anode and the electrolyte respectively. In the device, an open circuit voltage up to hundreds of millivolts is generated and a short-circuit discharging electric current reaches the microampere level. Interestingly, this 'battery' is rechargeable and can be turned on and off repeatedly without affecting the open circuit voltage. Furthermore, the demonstrated energy generator is capable of powering personal electronics. strongly broadened for some defects, other emitters possessed linewidths as narrow as $3 \mathrm{~nm}$. To further increase the brightness of the photon sources, Zhou and co-workers pre-patterned the sapphire substrate with cone-like cavities prior to the $\mathrm{GaN}$ deposition. This increased the extraction efficiency and yielded emission with megahertz rates. The reported solid-state SPEs can be easily integrated with on-chip quantum devices.

\section{ARTIFICIAL ORGANELLES \\ Sensing cellular cues}

Nat. Commun. 9, 1127 (2018)

Polymersomes are polymeric vesicles engineered to perform specific reactions in living cells, acting as simplified natural organelles. Similarly to their biological counterparts, they should be selectively permeable and responsive to specific cellular signals. While the first requirement is satisfied by inclusion of membrane proteins, channel porins or pore-forming peptides in the membrane, regulation of the polymersome activity through sensing of a cellular stimulus is challenging.

Now, Einfalt and co-workers synthesize a polymersome responsive to the intracellular concentration of glutathione (GSH). The responsive element is a genetically engineered channel porin containing two cysteine residues spatially close to the channel opening. Once inserted in the membrane, the porin regulates access to the polymersome on formation of a redoxsensitive complex with capping molecules. In oxidising conditions, the caps form disulphide bonds with the porin cysteines, blocking the entrance to the membrane channel, while in reducing conditions, the channel remains open. To demonstrate in vitro functionality, the authors encapsulate horse radish peroxidase within the vesicle, and measure enzymatic activity only in the presence of GSH.

The authors also demonstrate that their vesicles retain their redox-responsive activity in HeLa cells and in zebrafish embryo macrophages, with no sign of acute toxicity. The latter results support the possibility of using these artificial organelles for medical applications in vivo. 